\title{
Understanding the Role of Elastic Modulus in the Relationship Between Second Harmonic Generation Scattering and Tumor Cell Motility
}

\section{Tresa M. Elias}

University of Rochester

Danielle E. Desa

University of Rochester

Phong K. Nguyen

University of Rochester

Ananya Goyal

University of Rochester

Danielle Benoit

University of Rochester

Mark R. Buckley

University of Rochester

Catherine K. Kuo

University of Rochester David and Ilene Flaum Eye Institute

Edward Brown ( $\nabla$ edward_brown@urmc.rochester.edu )

University of Rochester https://orcid.org/0000-0002-5367-5330

\section{Research article}

Keywords: Second Harmonic Generation, SHG, F/B ratio, collagen, metastasis

Posted Date: September 9th, 2020

DOI: https://doi.org/10.21203/rs.2.23511/v2

License: (c) (1) This work is licensed under a Creative Commons Attribution 4.0 International License.

Read Full License 


\section{Abstract}

Background Second harmonic generation (SHG) is an intrinsic optical property of fibrillar collagen. SHG directionality is quantified by $\mathrm{F} / \mathrm{B}$, the ratio of forward- to backward-propagating signal, which is affected by collagen fiber internal structure, specifically the diameter, spacing, and disorder of fibrils within a collagen fiber. We have previously shown that F/B of primary invasive ductal carcinoma sections is prognostic of metastatic outcome. One possible cause of this relationship was revealed by our observation that tumor cells' motility on collagen I gels varied when collagen fiber internal structure in those gels was manipulated. The mechanism by which tumor cells sense changes in collagen fiber internal structure remains unknown: here we evaluate the role of elastic modulus in the relationship between collagen fiber internal structure (as reported by F/B) and tumor cell motility. Methods The 4T1 murine mammary adenocarcinoma, a model of metastatic triple negative breast cancer, (TNBC) and the 67NR line, a non-metastatic luminal phenotype, were introduced to a series of collagen-polyacrylamide mixed gels wherein collagen fiber internal structure and gel elastic modulus were independently controlled. F/B was measured with SHG, while elastic modulus was measured globally via rheometry and locally via atomic force microscopy (AFM). Tumor cell motility was quantified over three hours. Results The motility of both cell lines varied with F/B while elastic modulus was held constant, and did so at two physiological modulus values. Interestingly, 4T1 cell motility increased as F/B increased, while 67NR cell motility decreased. Conclusions Our results suggest that elastic modulus does not play a significant role in the observed relationship between collagen fiber internal structure (as reported by F/B) and tumor cell motility, for two cell lines that are models of TNBC and luminal-like breast cancer. Our observation that the two lines exhibit opposite motility trends as F/B increases is consistent with the trends in metastatic outcome versus $\mathrm{F} / \mathrm{B}$ observed in our published clinical data for luminal versus basal cohorts, and suggests that a tumor's subtype may play a role in their response to collagen fiber internal structure.

\section{Background}

Breast cancer is one of the most common cancers with approximately $12.8 \%$ of women being diagnosed within their lifetime (1). Between 2009 and 2015, women diagnosed with localized, or stage 1, breast cancer had a 98.8\% 5-year survival rate (1). However, women diagnosed with distant metastases had a significantly lower 5 -year survival rate of $27.4 \%$ (1). Metastasis to a secondary location is an onerous journey for a cancer cell. Once the primary tumor is established, cells begin to migrate within their microenvironment and may reach a blood or lymphatic vessel. Once a cell reaches the vessel it can intravasate into the lumen and, if it survives, reach a distant organ where it may lodge and/or extravasate and possibly develop into a metastasis (2).

One of the earliest steps in the metastatic process is migration through the tumor extracellular matrix (ECM). The ECM is made up of various proteins including fibrillar collagen type I, which can be imaged with an intrinsic optical signal caused by the scattering phenomenon known as second-harmonic generation (SHG)(3-5). SHG is observed when two incoming photons scatter off a noncentrosymmetric structure to produce one photon at twice the energy and half the wavelength of the individual incoming 
photons. In tumors the only significant source of SHG signal is fibrillar collagen (6). SHG imaging has revealed that tumor cells can move along collagen fibers, and that the orientation of collagen fibers with respect to the border between breast tumor and host tissue can predict patient outcomes (7). This suggests that collagen fibers may act as 'roads' facilitating tumor cell escape from the primary tumor. When an excitation laser is focused on one point along an individual collagen fiber, SHG photons can scatter in the "forwards" direction, i.e. the direction of propagation of the laser, or in the "backwards" direction, i.e. in the opposite direction. This directionality can be quantified by collecting photons in both directions using two detectors and calculating the forwards-to-backwards scattering ratio, or " $\mathrm{F} / \mathrm{B}$ ". $\mathrm{F} / \mathrm{B}$ is affected by properties of the internal structure of the collagen fiber including the diameter and spacing of the component fibrils that make up a fiber, as well as the order versus disorder in fibril packing (8-10). This internal structure is not the same as the direction of the fiber, but to extend the 'roads' analogy, F/B may provide information about road quality, as distinct from road direction.

Previously, we have shown that the F/B ratio of primary invasive ductal carcinoma (IDC) tumor biopsies is an independent prognostic indicator of patients' metastasis-free survival time in 10-year follow-up data (11). This suggests that F/B may be an additional indicator to assist in identifying which patients will metastasize and therefore who requires adjuvant chemotherapy. Hence the predictive ability of the F/B ratio of primary tumors may be useful in reducing the recognized clinical problem of "overtreatment"(12). To begin to understand why collagen fiber internal structure predicts metastatic outcome, in a previous study we determined the effect of varying collagen fiber internal structure on tumor cell motility in pure collagen gels (13). Collagen fiber internal structure was varied by varying gel polymerization conditions, and reported with F/B. We found that tumor cell motility was statistically significantly affected by varying collagen fiber internal structure (i.e. varying $F / B$ ) (13). This suggested that in patients, excised tumor F/B may be prognostic of metastatic outcome because the collagen fiber internal structure which dictates F/B also affects the efficiency with which cells escape the tumor. However, the underlying mechanisms of the relationship are still unidentified.

The elastic modulus of the extracellular matrix is known to have an impact on tumor cell behavior, including cell motility $(14,15)$. Therefore we hypothesized that cells could be reacting to modifications of collagen fiber internal structure because they are sensing matrix elastic modulus changes that may result from those modifications (15) . To test this hypothesis, herein we characterized the motility of tumor cell lines in a series of collagen/polyacrylamide mixed gels in which we independently varied the collagen fiber internal structure and the elastic modulus (16-18). These gels provide an in vitro environment to study tumor cell motility and its relationship to ECM properties in the absence of other cellular actors, and their secreted factors, that may be present in an in vivo model.

Our previous clinical study revealed interesting subtype-specific behavior: in the cohort with $\mathrm{ER}^{+}$ IDC tumors (i.e. including the luminal subtypes) patients whose tumors had higher F/B experienced fewer distant metastases in 10-year followup data (11). Conversely, in the cohort with ER' IDC tumors (i.e. including the TNBC and HER2 overexpressing subtypes) the relationship was inverted and patients whose tumors had lower F/B experienced fewer distant metastases. Therefore, in this study two cell lines were 
chosen: the metastatic 4T1 and nonmetastatic $67 \mathrm{NR}$ lines. The 4T1 murine mammary adenocarcinoma cells are a common model of basal-like TNBC and were used in our previous studies $(19,20)$. For the nonmetastatic cell line, 67NR was chosen since it is a paired sister cell line to the $4 \mathrm{~T} 1$ cells (i.e. derived from the same tumor) and has a contrasting luminal phenotype $(21,22)$.

\section{Methods}

\subsection{Multiphoton Image Acquisition}

Multiphoton laser scanning microscopy (MPLSM) was conducted using a Ti:Sapphire excitation laser controlled through a BX61WI upright microscope (Olympus, Shinjuku, Tokyo), with beam scanning and image acquisition controlled by an Olympus Fluoview FV300 scanning system. The excitation light was circularly polarized with $100 \mathrm{fs}$ pulses at $80 \mathrm{MHz}$. Circular polarization at the sample was achieved by passing the excitation light through a Berek compensator (Model 5540, New Focus, Irvine, CA) before entering the scan box, and verified as circular entering the objective lens with a Thorlabs PAX5710 Polarimeter. The light was focused through an Olympus UMPLFL20XW water immersion lens (20x, 0.95 N.A.), which was also used to collect the backwards scattered SHG signal. A $670 \mathrm{~nm}$ dichroic mirror (670 DCSP-2P, Chroma) was used to separate the backscattered signal from the excitation light. The backscattered SHG signal was filtered by a $405 \mathrm{~nm}$ band-pass filter (HQ405/30m-2P, Chroma). Forward SHG signal was captured in the forward-scattered direction using an Olympus 0.9 N.A. optical condenser, reflected by a $565 \mathrm{~nm}$ long-pass dichroic mirror (565 DCSX, Chroma), and filtered by a $405 \mathrm{~nm}$ band-pass filter (HQ405/30m-2P, Chroma). The SHG signal was generated with an excitation wavelength of $810 \mathrm{~nm}$. All signals were captured by Hamamatsu HC125-02 photomultiplier tubes.

\section{$2.2 \quad$ Cell Culture}

The 4T1 mouse mammary adenocarcinoma cell line (Caliper Life Sciences, Hopkinton, MA) was frozen after three initial passages. Cells were maintained in a media consisting of RPMI (Gibco, Invitrogen Inc., Carlsbad, CA), 10\% fetal bovine serum, and 1\% penicillin/streptomycin. 67NR cells were purchased from the Animal Model \& Therapeutic Evaluation Core (AMTEC) Barbara Karmanos Cancer Institute, Wayne State University. The $67 \mathrm{NR}$ cell line is a sister cell population to the $4 \mathrm{~T} 1$ cell line, both derived from a single spontaneous $\mathrm{Balb} / \mathrm{cfC}_{3} \mathrm{H}$. Cells were frozen after three initial passages. Cells were maintained in a media consisting of Dulbecco's Modified Eagle Medium (DMEM, Gibco 11965092), 10\% fetal bovine serum, 1\% 2mM L-glutamine (Cellgro 25-005-Cl), and 0.1\% 1mM Mixed Nonessential Amino Acids (Gibco 11140).

\subsection{Preparation of Collagen Polyacrylamide Mixed Gels}


Mattek petri dishes (Ashland, MA) with 14-mm diameter coverslip-bottomed wells were coated with $2.5 \mathrm{M} \mathrm{NaOH}$ and allowed to air dry. $200 \mu \mathrm{L}$ of $97 \%$ 3-aminopropyltrimethoxy silane (APTES) was added to the coverslip bottom for 5 minutes and then rinsed three times with distilled water and gently dried with Kim Wipes. Each dish was flooded with $0.5 \%$ glutaraldehyde for 30 minutes and then rinsed three times with distilled water and allowed to air dry. Separate $12 \mathrm{~mm}$ glass coverslips were treated with RainX and allowed to air dry. Each coverslip was wiped to ensure an even coating and then sprayed again.

Collagen gels were made of human type I collagen solution at a concentration of $3 \mathrm{mg} / \mathrm{mL}$ (Advanced Biomatrix, San Diego, CA). With all solutions kept at $4^{\circ} \mathrm{C}$ to minimize gelation, $810 \mu \mathrm{L}$ of collagen type I was combined in a conical tube with $108 \mu \mathrm{L}$ of RPMI 1640 media, and $50 \mu \mathrm{L}$ of distilled water. The solution was vortexed at the lowest speed for 5 seconds to ensure the solution was evenly mixed. The $\mathrm{pH}$ of the solution was measured with a Mettler Toledo Micro Pro $\mathrm{pH}$ meter (Columbus, $\mathrm{OH}$ ). The acceptable $\mathrm{pH}$ ranges for this solution is discussed below. In a petri dish, 6 droplets of $125 \mu \mathrm{L}$ each of the solution was pipetted, making sure the droplets did not touch or spread and that all droplets were equal in size to ensure equal gelation. The petri dish was placed in an incubator for 2 hours at $37^{\circ} \mathrm{C}$ and $5 \% \mathrm{CO}_{2}$.

Polyacrylamide is an easily manipulated polymer that can produce a wide range of elastic moduli, and polyacrylamide hydrogels do not significantly interact with the cell surface $(16,23,24)$. When mixed with collagen the polyacrylamide can independently influence overall gel elastic modulus (16-18). Two polyacrylamide precursor solutions were made with different concentrations of acrylamide and bisacrylamide. The first solution was $5 \%$ acrylamide: $0.1 \%$ bis-acrylamide and the second solution was $12 \%$ acrylamide: $0.25 \%$ bis-acrylamide; these combinations are known to produce polyacrylamide gels of significantly different elastic moduli (17). $375 \mu \mathrm{L}$ of the desired polyacrylamide precursor was added to a conical tube. Once the pure collagen gel droplets gelled, they were added to the polyacrylamide precursor solution. This solution was vortexed on medium speed for 20 seconds to break up the collagen gels and mix thoroughly with the polyacrylamide. Next, $0.5 \mu \mathrm{L}$ of $\mathrm{N}, \mathrm{N}, \mathrm{N}^{\prime}, \mathrm{N}^{\prime}$-tetramethylethylenediamine was added to the solution and followed by $5 \mu \mathrm{L}$ of $10 \% \mathrm{w} / \mathrm{v}$ ammonium persulfate, for a final concentration of $0.5 \%$ and $5 \%$, respectively; to minimize premature polymerization, this step was completed within 2 minutes. The solution was mixed by pipetting gently 10 times while avoiding bubbles. $100 \mu \mathrm{L}$ of the solution was pipetted onto the center of the treated square coverslip, and then coverslipped with the treated circular coverslip. The solution was left at room temperature for 30 minutes to fully polymerize. This produces a final mixed gel with a uniform distribution of collagen fibers visible in SHG (Figure 1) embedded within a polyacrylamide matrix. The final mixed gels had a denser network of fibers than contained in the precursor pure collagen gels that were added to polyacrylamide precursor and vortexed.

The top coverslip was carefully removed with tweezers and the dish was washed with phosphate-buffered saline (PBS). The PBS was replaced with either RPMI or DMEM media, depending on which cells would be added to the gels. Gels were stored in the incubator overnight and subsequent experiments were performed within 24 hours. 


\subsection{Determining the F/B Ratio of Collagen Polyacrylamide Mixed Gels}

During each imaging session, a standard fluorescein isothiocyanate (FITC) calibration sample was imaged with an excitation wavelength of $810 \mathrm{~nm}$ and $535 \mathrm{~nm}$ emission filters. Free FITC emits isotopically, which means the F/B ratio of a FITC calibration sample should be 1.0; any variation in the imaging system is reported by a variation in this measured ratio. To account for any day-to-day variation in the system, the background-subtracted SHG F/B ratios of the imaged samples on a given day (discussed below) are divided by the background-subtracted F/B ratio of the FITC calibration taken on that day. For each image pair, an F and B image was taken with the laser shutter closed and the average pixel counts for each image provided the background.

For SHG F/B ratio analysis of the gels, two xyz stacks of simultaneously collected forward and backwards images were taken at a given xy region on the gel; three different regions of each gel were imaged. The forward and backward image pairs were taken at $50 \mu \mathrm{m}$ steps through the depth of the gel. Each image was $660 \mu \mathrm{m} \times 660 \mu \mathrm{m}$.

ImageJ was used for all image analysis (25). The forward and backward images were background subtracted with the average background value for the corresponding direction. For each matched image pair, two masks of both the forward and the backward-scattered SHG signals were created, in which all collagen pixels were set to 1 and background pixels were set to 0 by applying a predetermined threshold to each of the two images. One forward threshold and one backward threshold was used for each imaging session and applied to all images. These thresholds were determined by surveying random background-subtracted images and adjusting the image's look-up tables until only collagen fibers are highlighted, and all other pixels were rejected. The two resultant masks for each matched image pair were then multiplied together to create a single mask that identifies pixels with SHG emission above the threshold in both the forward and backwards direction. A single F/B ratio image was calculated from the $\mathrm{F}$ and $\mathrm{B}$ image pair and then multiplied by the mask to set all non-fiber pixels to zero, thereby excluding these pixels from the analysis. The average pixel value of fiber pixels (i.e. non-zero pixels) for that region was then calculated for the masked F/B ratio image. This process was repeated in a for loop for each image pair in the stack for a single region. The average pixel value of each slice in the stack was then averaged to give a single F/B ratio for that region of the gel. The F/B ratio from all three regions were then averaged to produce a single $\mathrm{F} / \mathrm{B}$ ratio to represent the entire gel.

\subsection{Manipulation of F/B Ratio}

The $\mathrm{F} / \mathrm{B}$ ratio of the gels was altered by manipulating the $\mathrm{pH}$ of the collagen gel in the initial stage of making the mixed collagen-polyacrylamide gels as previously described (13). This is known to vary fibril diameter $(26,27)$, one element of collagen fiber internal structure known to alter $F / B(13)$ 
(others being the less experimentally tractable fibril spacing and order versus disorder of fibril packing into fibers) $(9,10)$. The $\mathrm{pH}$ was altered by using increasing amounts of $2.5 \mathrm{M} \mathrm{NaOH}$ in the collagen gel solution to bring the final pH to 7.5-7.7, 8.5 - 8.7, or 9.5 - 9.7, measured with a Mettler Toledo Micro Pro $\mathrm{pH}$ meter (Columbus, $\mathrm{OH}$ ). The collagen gels were gelled and incorporated into the polyacrylamide as stated above.

\subsection{Analysis of Tumor Cell Motility}

The following preparation and imaging were performed one gel at a time. An hour prior to imaging, all media was removed from the gel and $1 \times 10^{4}$ cells were resuspended in their respective media and added to the surface of the gel in a droplet. The gel was placed in the incubator for an hour (28) to allow the cells to adhere to the surface before all excess media was removed to minimize motion during the imaging session. In a preliminary experiment, representative gels were made with and without the addition of collagen, and we found that after the standard one hour adherence time, a gentle rinse washed away all cells in the collagen-free cases while the seeded cells remained adhered to the gel in the collagen-including cases (data not shown). This verifies that the cells were indeed interacting with the collagen versus the nonreactive polyacrylamide. Live cell images were taken on a Nikon Eclipse Ti microscope, using a Nikon MRH201201 air lens (20x, 0.45 N.A.). Cells were quickly transferred to a microscope stage incubator (Pathology Devices, Westminster, $\mathrm{MD}$ ), at $37^{\circ} \mathrm{C}, 5 \% \mathrm{CO}_{2}$, and $85 \%$ relative humidity. One phase contrast image was taken every 2 minutes for 3 hours and saved as a tiff file. To assess the extent of adhesion we quantified the aspect ratio of each cell on the first image of the threehour timelapse (i.e. after the one-hour adhesion time) as well as the last image of the timelapse, and found no statistical differences within any group as well as no main effect of gel F/B or elastic modulus (2-way ANOVA with Bonferroni post-hoc tests, Supplemental Figure 1). This suggests that the initial adhesion time was sufficient for cells to fully adhere.

All image analysis was performed in ImageJ (25). For each gel, every cell that remained isolated in the time series (i.e. not touching another cell for more than 3 frames) was tracked using the "Manual Tracking" ImageJ plugin, which tabulates the XY location of each cell at each time point (25). From this data, the total distance traveled was calculated for each cell by summing the distance traveled between each time point. To confirm that the gels did not significantly move during the imaging session, a fiducial artifact on each gel was identified and tracked in the same manner; total distance traveled by fiducial artifacts did not exceed $4.37 \mu \mathrm{m}$ per imaging session and averaged $2.17 \mu \mathrm{m} \pm 1.62 \mu \mathrm{m}$, significantly less than the motion of all cells in all conditions. For comparison with previous literature (13), imaging sessions did not exceed three hours to ensure that there was no significant penetration of the cells into the gels and hence that the analysis remained two-dimensional, as well as to ensure that the cells themselves did not significantly manipulate F/B of the gel. 
A silicon nitride tip probe with a radius of $20 \mathrm{~nm}$ and spring constant of $0.06 \mathrm{~N} / \mathrm{m}$ (Bruker, CA, USA) was used on an MFP-3D AFM (Asylum Research, CA, USA) to obtain a 64 x 64 two-dimensional force map over a $20 \mu \mathrm{m} \times 20 \mu \mathrm{m}$ area in the center of gels that were $600-700 \mu \mathrm{m}$ thick. Elastic modulus measurements at each point on the force map were calculated by fitting force displacements curves to the Hertzian model, and force maps were averaged to yield a single elastic modulus measurement for each gel, as we previously described(29-32).

\subsection{Measuring Elastic Modulus with Rheology}

Global elastic modulus measurements of $8 \mathrm{~mm}$ diameter gels were performed using a parallel plate torsional rheometer (Discovery HR-2, TA Instruments). To prevent slippage, the gap distance between the rheometer plate and base was reduced in small steps $(\sim 10 \mathrm{~mm})$ until the specimen was compressed with a constant axial force of $-0.3 \mathrm{~N}$. After compression, dynamic torsional shear mechanical tests were performed at an angular frequency of $\omega=1 \mathrm{rad} / \mathrm{s}$ across a range of strain amplitudes $(0.01-1 \%)$. The shear storage modulus $\left(G^{\prime}\right)$ was then computed at each strain level based on the measured timedependent torque and angular displacement of the top plate. The value of $\mathrm{G}^{\prime}$ for each gel was taken to be the average measurement of $\mathrm{G}^{\prime}$ across all strain amplitudes. Assuming the gels were isotropic, the elastic modulus $\mathrm{E}$ was then determined according to $\mathrm{E}=3 \mathrm{G}^{\prime}$.

\section{Results}

3.1 Development of Consistent Mixed Collagen/Polyacrylamide Gels that Maintain Optical Properties Necessary for MPLSM and Have Tunable Physical Properties

A series of gels were produced over a range of $\mathrm{F} / \mathrm{B}$ and elastic modulus values. Figure 1 shows representative forward-scattered SHG images of one image plane close to the surface of one gel from each condition, revealing no significant visible change in gel organization with F/B or elastic modulus.

To obtain a single representative F/B value for a gel, the sample being imaged must be optically thin, which means that the measured F/B must not be affected by the imaging depth into the sample. This is accomplished either with a sample that is not very scattering, is not very thick, or both. When plotting F/B versus imaging depth, non-optically thin samples would have a slope across the gel depth which was significantly different than zero. The methods described above produced small mixed collagen/polyacrylamide gels whose F/B value did not follow a significant trend as a function of depth within the gel (Figure 2). This was verified for each gel by plotting F/B as a function of imaging depth and performing a linear regression (Supplemental Figure 2), and in each gel tested the slope of the line of best fit was not significantly different from zero, thus ensuring that the measured F/B ratio was not 
significantly affected by imaging depth. To avoid including F/B of pixels outside the gel in that screening process, the slope of the middle $50 \%$ of the stack was analyzed.

\subsection{Manipulation of F/B Ratio and Confirmation that F/B was Not Affected by Addition of Polyacrylamide}

The $\mathrm{F} / \mathrm{B}$ ratio of the gels was manipulated by altering the $\mathrm{pH}$ of the collagen gel during fibrillogenesis, and elastic modulus was manipulated by altering polyacrylamide polymerization, each as described above. The $\mathrm{pH}$ of the collagen was adjusted to either $7.5,8.5$ or 9.5. Figure 3 shows that $\mathrm{F} / \mathrm{B}$ increases as $\mathrm{pH}$ of the collagen during synthesis increases, which is consistent with our previous studies (13). A twoway ANOVA was performed to compare the F/B of the high and low elastic modulus gels within a single $\mathrm{pH}$ category, and revealed no effect of elastic modulus $(p=0.24)$. A Bonferroni post-hoc test revealed that there was no statistically significant difference between the F/B of the high and low modulus gels within each $\mathrm{pH}$ category. The range of $\mathrm{F} / \mathrm{B}$ values that result from these manipulations ( 6 to $\sim 11)$ is consistent with the range of values found in tumor tissue measured on the same system $(11,13)$.

\subsection{Evaluation of Elastic Modulus of Gels}

The elastic modulus of the gels was manipulated by altering the acrylamide and bis-acrylamide concentrations in the polyacrylamide precursor solution. The 'high modulus' gels were composed of $12 \%$ acrylamide and $0.25 \%$ bis-acrylamide and the 'low modulus' gels were composed of $5 \%$ acrylamide and $0.1 \%$ bis-acrylamide. Figure 4 shows the elastic modulus of gels produced with these procedures, as measured by both AFM and rheology. In each modulus category (i.e. low and high), a two-way ANOVA was performed with Bonferroni post-hoc test using $\mathrm{pH}$ category and modulus measurement method as independent variables. No significant effect of $\mathrm{pH}$ category $(\mathrm{p}=0.79)$ nor of method of modulus measurement was found $(\mathrm{p}=0.66)$, nor did Bonferroni testing find differences between any measured values of modulus within each combination of $\mathrm{pH} / \mathrm{modulus}$ category. This reveals that the tuning of $\mathrm{F} / \mathrm{B}$ by varying $\mathrm{pH}$ during collagen gel formation does not affect gel modulus, and that the two methods of measuring elastic modulus give the same results. Taken together this data demonstrates that the mixed gels had an elastic modulus that could be tuned between two desired values (confirmed with both bulk rheology measurements as well as local AFM measurements), that the average values of elastic modulus were $1.738 \pm 0.168 \mathrm{kPa}$ and $18.504 \pm 0.685 \mathrm{kPa}$ (values that are relevant to tumor physiology (33)), and that alteration of collagen gelation did not affect the elastic modulus of the mixed gel.

\section{4 $\quad 4 T 1$ and 67NR Cell Motility}


Tumor cells moved about on the gels in typical fashion, with some cells appearing "stretched out" while others remained somewhat more rounded and moved about with a visibly "active" edge (See Supplemental Videos). Typical motility tracks are shown in Figure 5 and range from small motions in the general vicinity of the starting point to longer excursions. Overall, the histogram of distances traveled revealed a roughly Gaussian distribution without any marked secondary peaks indicative of a highly mobile or immobile fraction (Supplemental Figure 3).

Figures 6 and 7 shows the effect of varying collagen microstructure and elastic modulus on tumor cell motility of $4 \mathrm{~T} 1$ cells and $67 \mathrm{NR}$ cells, while Table 1 provides the number of cells analyzed for each condition. For 4T1 cells (Figure 6), 2-way ANOVA reveals an effect of elastic modulus $(p<0.0001)$ and an effect of $F / B(p=0.014)$ on total distance traveled, but no interaction $(p=0.85)$. Bonferroni posthoc testing reveals that the total distance traveled by the three low modulus groups are individually different from the three high modulus groups ( $p<0.05,0.05$, and 0.01 , respectively). This demonstrates that the motility of $4 \mathrm{~T} 1$ cells can respond to variations in collagen fiber internal structure (as reported by F/B) independently of elastic modulus.

For 67NR cells (Figure 7), 2-way ANOVA reveals an effect of F/B ( $p=0.0015)$ but no effect of elastic modulus $(p=0.99)$ on total distance traveled, and no interaction $(p=0.31)$. Bonferroni post-hoc testing reveals no differences between the total distance traveled by the low and high modulus groups at any of the three F/B conditions. Overall, this demonstrates that the motility of 67NR cells can respond to variations in collagen fiber internal structure (as reported by F/B) independently of elastic modulus. Interestingly, the trend for the most motile 67NR cells is negative (i.e. the slope of the relationship between distance traveled versus F/B is negative), whereas the trend is positive for the $4 T 1$ cells.

\section{Discussion}

To evaluate the role of elastic modulus in the relationship between collagen fiber internal structure, as reported by $F / B$, and tumor cell motility, we introduced cell lines to mixed collagen/polyacrylamide gels with three physiologically relevant $F / B$ values and two physiologically relevant values of elastic modulus. First, we created a series of in vitro gels with multiple values of F/B and elastic modulus. To develop these gels in our laboratory, we incorporated polyacrylamide into our previous pure collagen gel protocols (13). After developing this protocol, we first verified that the visible morphology of the collagen fibrils did not vary when F/B and elastic modulus were tuned (Figure 1). Next, we verified that the measured F/B was constant throughout each gel and was not affected by the presence of the polyacrylamide nor by variation of the elastic modulus (Figure 3, Supplementary Figure 2). Next, we confirmed that varying collagen fiber internal structure (as reported by F/B) by varying collagen polymerization conditions did not affect the measured elastic modulus and that all three F/B categories had the same measured elastic modulus within each of two modulus categories (Figure 4).

As demonstrated in Figure 6, in mixed collagen-polyacrylamide gels the motility of 4T1 cells increases as F/B of their microenvironment increases. These results confirm our previous study where we observed 
4T1 motility increasing as F/B increased in pure collagen gels whose elastic modulus was not controlled (13). Furthermore, this specific trend of tumor cell motility increasing with $F / B$ is consistent with the trend of metastasis-free survival time decreasing with $\mathrm{F} / \mathrm{B}$ observed in patient tumor samples in the analogous (ER', containing basal TNBC) cohort of human patients. This suggests that $F / B$ may predict metastatic outcome in patient samples because changing collagen fiber internal structure (which affects $F / B$ ) alters the outflow of cells from a tumor. Importantly, 4T1 motility increased with F/B independently of elastic modulus. This suggests that the 4T1 model of TNBC can respond to changes in internal collagen structure through some other mechanism. In the 67NR model of luminal breast cancer we again observed that tumor cell motility varies with F/B and does so independently of elastic modulus (Figure 7). This suggests that the 67NR model of luminal breast cancer can also respond to changes in internal collagen structure through some other mechanism. Interestingly, we observe the opposite trend between motility and F/B in the 67NR cells versus 4T1 cells, where 67NR tumor cell motility decreases as F/B of the microenvironment increases. This is again consistent with the data from patient samples where metastasis-free survival time increases with increasing $F / B$ in the $E R+$ patient cohort, which contains the luminal subtypes (11). This again suggests that $F / B$ may predict metastatic outcome in patient samples because changing collagen fiber internal structure (which affects $F / B$ ) alters the outflow of cells from a tumor. However it appears that the response of luminal-type breast tumor cells to alterations in collagen internal structure is opposite to the response of basal-type TNBC breast tumor cells (Figures 6 and 7), although in both cases the response is independent of elastic modulus, but directly to changes in the collagen fiber internal structure.

The mechanisms by which these two cell lines respond to tuning of collagen fiber internal structure independently of elastic modulus remain unknown. There are myriad differences between the two lines, just as there are between patient breast tumors of luminal and basal subtypes, including in their metabolism, overall gene expression patterns, etc. $(34,35)$. While ER, PR, and HER2 status are useful markers to distinguish these subtypes we do not have any evidence that those particular receptors are involved. One attractive candidate for sensing the variation in fibril diameter that is induced by varying $\mathrm{pH}$ during collagen gel synthesis (hence tuning F/B) is the discoidin domain receptor (DDR) family, which are collagen sensors known to be sensitive to the morphological state of collagen fibers (36), and whose activity is critical for breast cancer metastasis and tumor collagen organization (37).

This work also confirms observations in the literature that the motility of tumor cells varies when elastic modulus of their substrate is varied, and that different cell lines respond to changes in elastic modulus in different ways. At equivalent values of elastic modulus and F/B we observed that $4 \mathrm{~T} 1$ cells were more motile than 67NR cells (Figure 6 and 7). This is consistent with literature observations of their relative migration (38) and with the fact that unlike the highly metastatic $4 \mathrm{~T} 1$ cells, $67 \mathrm{NR}$ cells very rarely metastasize to secondary locations and are typically unable to leave the primary site (21). At constant values of F/B the motility of $4 T 1$ cells increase with elastic modulus while the motility of 67NR cells do not change (Figure 6 and 7). This is consistent with literature observations of MDA-MB-231 (a model of basal TNBC cancer, analogous to 4T1s) and MCF7 (a model of luminal breast cancer, analogous to 
67NRs) which exhibited the exact same behavior (i.e. increasing motility versus no change in motility) on substrates of similar elastic modulus (39).

There are several limitations to this work. While we do not observe any significant variations in visible gel morphology when F/B and elastic modulus are tuned (Figure 1), there may be some gel property that we are unexpectedly varying that is not visible in our images. Also, while AFM offers a well-established approach to test the mechanical properties of tissues at the cellular length scale $(32,40)$, the pressing of a nanoscale AFM tip on a collagen fibril embedded in a network of collagen and polyacrylamide is a somewhat different interaction than the tugging of a nanoscale cell filopodia on that fibril. Hence, while the AFM and rheometer measurements suggest that the nanoscale and macroscale elastic modulus of the gel network is constant as F/B is varied, there is a possibility that the cells can sense mechanical differences in the fibrils not apparent to nanoscale AFM tips, as encompassed by the difference in AFM/fibril versus cell/fibril interactions.

\section{Conclusions}

To summarize, using a collagen/polyacrylamide gel system capable of independently varying collagen fiber internal structure and elastic modulus, we evaluated tumor cell motility under the influence of these two parameters. Our results suggest that variation in collagen fiber internal structure (as reported by F/B) can induce variation in tumor cell motility directly, independently of elastic modulus, in either a model of basal-like metastatic TNBC or a model of luminal-like non-metastatic breast cancer. Furthermore, we found that in the 67NR model of luminal breast cancer cell motility decreases as F/B increases, while in the 4T1 model of basal TNBC cell motility increases with F/B. This is consistent with observations of patient samples wherein luminal subtypes exhibited longer metastasis free survival (MFS) with increasing F/B while basal subtypes exhibited shorter MFS with increasing F/B (22). Taken together these results suggest that variations in collagen fiber internal structure do not modify tumor cell motility via any induced changes in elastic modulus. Furthermore, these results suggest that luminal and basal-type breast cancer reacts to changes in collagen fiber internal structure in different ways. In future work we hope to continue to explore the mechanism by which tumor cells sense changes in collagen fiber internal structure. Our observation that these cells are not sensing changes in collagen fiber internal structure through elastic modulus suggests that there may be cell surface receptors that are directly sensitive to variations in this property. Furthermore, these fiber internal structure sensors may be different on basal versus luminal subtypes or may be linked to different signaling pathways. This increased understanding of the mechanisms relating $\mathrm{F} / \mathrm{B}$, collagen fiber internal structure, and metastasis, may improve our understanding of the metastatic process, possibly leading to improved prognostic tools or antimetastatic therapies.

\section{Abbreviations}

SHG: Second Harmonic Generation, F/B: Forward-to-backwards ratio, IDC: Invasive Ductal Carcinoma, ER+: Estrogen Receptor positive, ER-: Estrogen Receptor negative, ECM: Extracellular Matrix, MPLSM: 
Multiphoton Laser Scanning Microscopy, PBS: Phosphate Buffer Saline, FITC: Fluorescein Isothiocyanate, AFM: Atomic Force Microscopy, MFS: Metastasis-free Survival

\section{Declarations}

\section{Ethics approval and consent to participate}

Not applicable

\section{Consent for publication}

Not applicable

\section{Availability of data and materials}

The datasets used and/or analyzed during the current study are available from the corresponding author on reasonable request

\section{Competing interests}

The authors declare that they have no competing interests

\section{Funding}

This work was funded by Department of Defense Breast Cancer Research Program grant W81XWH-17-10011 and National Institutes of Health National Cancer Institute grant R21CA208921 to EB, Department of Defense Breast Cancer Research Program grant W81XWH-17-1-0015 to CKK, and a Wilmot Cancer Institute grant and a Breast Cancer Coalition of Rochester predoctoral traineeship to DD. The funding bodies had no role in in the design of the study, data collection, data analysis, data interpretation of data, nor in writing the manuscript.

\section{Authors' Contributions}

TE designed the protocol for collagen-polyacrylamide gels in consultation with PN and CKK, and prepared all gels for experiments, imaged and analyzed F/B data from all gels, performed cell motility experiments, and completed statistical analyses. TE is responsible for writing the majority of the manuscript. DD contributed to the design of the experiment as well as data interpretation and troubleshooting. AG performed all rheometer measurements, as well as analyzed elastic modulus data. PN performed all AFM elastic modulus measurements and contributed to AFM analysis. CK provided consultation on making collagen-PA gels, access to AFM, as well as expertise and advice involving AFM and motility results. DB provided access to live cell imaging microscope, cell culture hoods, and incubators. MB provided access to rheometer as well as expertise in analyzing and comparing elastic modulus results. EB contributed to the design of the experiments, troubleshooting, data interpretation, and writing the manuscript. 
All authors read and approved the final manuscript.

\section{Acknowledgements}

Not Applicable

\section{Authors' Information}

Not Applicable

\section{References}

1. National Cancer Institute Surveillance E, and End Results Program. Cancer Stat Facts: Female Breast Cancer: National Cancer Institute; 2019 [cited 2019 November 10th]. Available from: https://seer.cancer.gov/statfacts/html/breast.html

2. Jin X, Mu P. Targeting Breast Cancer Metastasis. Breast Cancer (Auckl). 2015;9(Suppl 1):23-34.

3. Zoumi A, Yeh A, Tromberg BJ. Imaging cells and extracellular matrix in vivo by using secondharmonic generation and two-photon excited fluorescence. Proc Natl Acad Sci U S A. 2002;99(17):11014-9.

4. Campagnola PJ, Millard AC, Terasaki M, Hoppe PE, Malone CJ, Mohler WA. Three-dimensional highresolution second-harmonic generation imaging of endogenous structural proteins in biological tissues. Biophys J. 2002;82(1 Pt 1):493-508.

5. Freund I, Deutsch M, Sprecher A. Connective tissue polarity. Optical second-harmonic microscopy, crossed-beam summation, and small-angle scattering in rat-tail tendon. Biophys J. 1986;50(4):693712.

6. Brown E, McKee T, diTomaso E, Pluen A, Seed B, Boucher Y, et al. Dynamic imaging of collagen and its modulation in tumors in vivo using second-harmonic generation. Nat Med. 2003;9(6):796-800.

7. Conklin MW, Eickhoff JC, Riching KM, Pehlke CA, Eliceiri KW, Provenzano PP, et al. Aligned collagen is a prognostic signature for survival in human breast carcinoma. Am J Pathol. 2011;178(3):1221-32.

8. Han X, Burke RM, Zettel ML, Tang P, Brown EB. Second harmonic properties of tumor collagen: determining the structural relationship between reactive stroma and healthy stroma. Opt Express. 2008;16(3):1846-59.

9. Williams RM, Zipfel WR, Webb WW. Interpreting second-harmonic generation images of collagen I fibrils. Biophys J. 2005;88(2):1377-86.

10. Lacomb R, Nadiarnykh O, Townsend SS, Campagnola PJ. Phase Matching considerations in Second Harmonic Generation from tissues: Effects on emission directionality, conversion efficiency and observed morphology. Optics communications. 2008;281(7):1823-32.

11. Burke K, Smid M, Dawes RP, Timmermans MA, Salzman P, van Deurzen $\mathrm{CH}$, et al. Using second harmonic generation to predict patient outcome in solid tumors. BMC Cancer. 2015;15:929. 
12. Weigelt B, Peterse JL, van 't Veer LJ. Breast cancer metastasis: markers and models. Nat Rev Cancer. 2005;5(8):591-602.

13. Burke KA, Dawes RP, Cheema MK, Van Hove A, Benoit DS, Perry SW, et al. Second-harmonic generation scattering directionality predicts tumor cell motility in collagen gels. J Biomed Opt. 2015;20(5):051024.

14. Kraning-Rush CM, Reinhart-King CA. Controlling matrix stiffness and topography for the study of tumor cell migration. Cell Adh Migr. 2012;6(3):274-9.

15. Lo CM, Wang HB, Dembo M, Wang YL. Cell movement is guided by the rigidity of the substrate. Biophys J. 2000;79(1):144-52.

16. Wang YL, Pelham RJ, Jr. Preparation of a flexible, porous polyacrylamide substrate for mechanical studies of cultured cells. Methods Enzymol. 1998;298:489-96.

17. Syed S, Karadaghy A, Zustiak S. Simple polyacrylamide-based multiwell stiffness assay for the study of stiffness-dependent cell responses. J Vis Exp. 2015(97).

18. Baek K, Clay NE, Qin EC, Sullivan KM, Kim DH, Kong H. In situ assembly of the collagenpolyacrylamide interpenetrating network hydrogel: Enabling decoupled control of stiffness and degree of swelling. European Polymer Journal. 2015;72:413-22.

19. Martin JL, Julovi SM, Lin MZ, de Silva HC, Boyle FM, Baxter RC. Inhibition of basal-like breast cancer growth by FTY720 in combination with epidermal growth factor receptor kinase blockade. Breast Cancer Res. 2017;19(1):90.

20. Ghosh A, Sarkar S, Banerjee S, Behbod F, Tawfik O, McGregor D, et al. MIND model for triple-negative breast cancer in syngeneic mice for quick and sequential progression analysis of lung metastasis. PLoS One. 2018;13(5):e0198143.

21. Aslakson CJ, Miller FR. Selective events in the metastatic process defined by analysis of the sequential dissemination of subpopulations of a mouse mammary tumor. Cancer Res. 1992;52(6):1399-405.

22. Johnstone CN, Smith YE, Cao Y, Burrows AD, Cross RS, Ling X, et al. Functional and molecular characterisation of E0771.LMB tumours, a new C57BL/6-mouse-derived model of spontaneously metastatic mammary cancer. Dis Model Mech. 2015;8(3):237-51.

23. Caliari SR, Burdick JA. A practical guide to hydrogels for cell culture. Nat Methods. 2016;13(5):40514.

24. Gilchrist CL, Darling EM, Chen J, Setton LA. Extracellular Matrix Ligand and Stiffness Modulate Immature Nucleus Pulposus Cell-Cell Interactions. PLoS One. 2011;6(11).

25. Rueden $C T$, Schindelin J, Hiner MC, DeZonia BE, Walter AE, Arena ET, et al. ImageJ2: ImageJ for the next generation of scientific image data. BMC Bioinformatics. 2017;18(1):529.

26. Roeder BA, Kokini K, Sturgis JE, Robinson JP, Voytik-Harbin SL. Tensile mechanical properties of three-dimensional type I collagen extracellular matrices with varied microstructure. J Biomech Eng. 2002;124(2):214-22. 
27. Wood GC, Keech MK. The formation of fibrils from collagen solutions. 1. The effect of experimental conditions: kinetic and electron-microscope studies. Biochem J. 1960;75(3):588-98.

28. Peyton SR, Putnam AJ. Extracellular matrix rigidity governs smooth muscle cell motility in a biphasic fashion. J Cell Physiol. 2005;204(1):198-209.

29. Pan XS, Li J, Brown EB, Kuo CK. Embryo movements regulate tendon mechanical property development. Philosophical transactions of the Royal Society of London Series B, Biological sciences. 2018;373(1759).

30. Marturano JE, Schiele NR, Schiller ZA, Galassi TV, Stoppato M, Kuo CK. Embryonically inspired scaffolds regulate tenogenically differentiating cells. J Biomech. 2016;49(14):3281-8.

31. Schiele NR, von Flotow F, Tochka ZL, Hockaday LA, Marturano JE, Thibodeau JJ, et al. Actin cytoskeleton contributes to the elastic modulus of embryonic tendon during early development. $J$ Orthop Res. 2015;33(6):874-81.

32. Marturano JE, Arena JD, Schiller ZA, Georgakoudi I, Kuo CK. Characterization of mechanical and biochemical properties of developing embryonic tendon. Proc Natl Acad Sci U S A. 2013;110(16):6370-5.

33. Plodinec M, Loparic M, Monnier CA, Obermann EC, Zanetti-Dallenbach R, Oertle P, et al. The nanomechanical signature of breast cancer. Nat Nanotechnol. 2012;7(11):757-65.

34. Xi Y, Shi J, Li W, Tanaka K, Allton KL, Richardson D, et al. Histone modification profiling in breast cancer cell lines highlights commonalities and differences among subtypes. BMC Genomics. 2018;19(1):150.

35. Simões RV, Serganova IS, Kruchevsky N, Leftin A, Shestov AA, Thaler HT, et al. Metabolic plasticity of metastatic breast cancer cells: adaptation to changes in the microenvironment. Neoplasia. 2015;17(8):671-84.

36. Yeung DA, Shanker N, Sohail A, Weiss BA, Wang C, Wellmerling J, et al. Clustering, Spatial Distribution, and Phosphorylation of Discoidin Domain Receptors 1 and 2 in Response to Soluble Collagen I. J Mol Biol. 2019;431(2):368-90.

37. Corsa CA, Brenot A, Grither WR, Van Hove S, Loza AJ, Zhang K, et al. The Action of Discoidin Domain Receptor 2 in Basal Tumor Cells and Stromal Cancer-Associated Fibroblasts Is Critical for Breast Cancer Metastasis. Cell Rep. 2016;15(11):2510-23.

38. Eckhardt BL, Parker BS, van Laar RK, Restall CM, Natoli AL, Tavaria MD, et al. Genomic analysis of a spontaneous model of breast cancer metastasis to bone reveals a role for the extracellular matrix. Mol Cancer Res. 2005;3(1):1-13.

39. Ansardamavandi A, Tafazzoli-Shadpour M, Shokrgozar MA. Behavioral remodeling of normal and cancerous epithelial cell lines with differing invasion potential induced by substrate elastic modulus. Cell Adh Migr. 2018;12(5):472-88.

40. Loparic M, Wirz D, Daniels AU, Raiteri R, Vanlandingham MR, Guex G, et al. Micro- and nanomechanical analysis of articular cartilage by indentation-type atomic force microscopy: validation with a gel-microfiber composite. Biophys J. 2010;98(11):2731-40. 


\section{Tables}

Table 1. Number of each cell type analyzed in each of three experimental replicates for each F/B and elastic modulus condition.

\begin{tabular}{|c|c|c|c|c|c|}
\hline \multicolumn{6}{|c|}{$67 \mathrm{NR}$} \\
\hline \multicolumn{3}{|c|}{ Low Modulus } & \multicolumn{3}{|c|}{ High Modulus } \\
\hline Low F/B & Medium F/B & High F/B & Low F/B & Medium F/B & High F/B \\
\hline 27 & 21 & 16 & 27 & 14 & 14 \\
\hline 10 & 12 & 10 & 24 & 15 & 10 \\
\hline 7 & 16 & 10 & 18 & 10 & 12 \\
\hline \multicolumn{6}{|c|}{$4 \mathrm{~T} 1$} \\
\hline \multicolumn{3}{|c|}{ Low Modulus } & \multicolumn{3}{|c|}{ High Modulus } \\
\hline Low F/B & Medium F/B & High F/B & Low F/B & Medium F/B & High F/B \\
\hline 10 & 16 & 22 & 25 & 21 & 27 \\
\hline 17 & 15 & 22 & 23 & 27 & 32 \\
\hline 13 & 21 & 21 & 32 & 27 & 30 \\
\hline
\end{tabular}

\section{Figures}




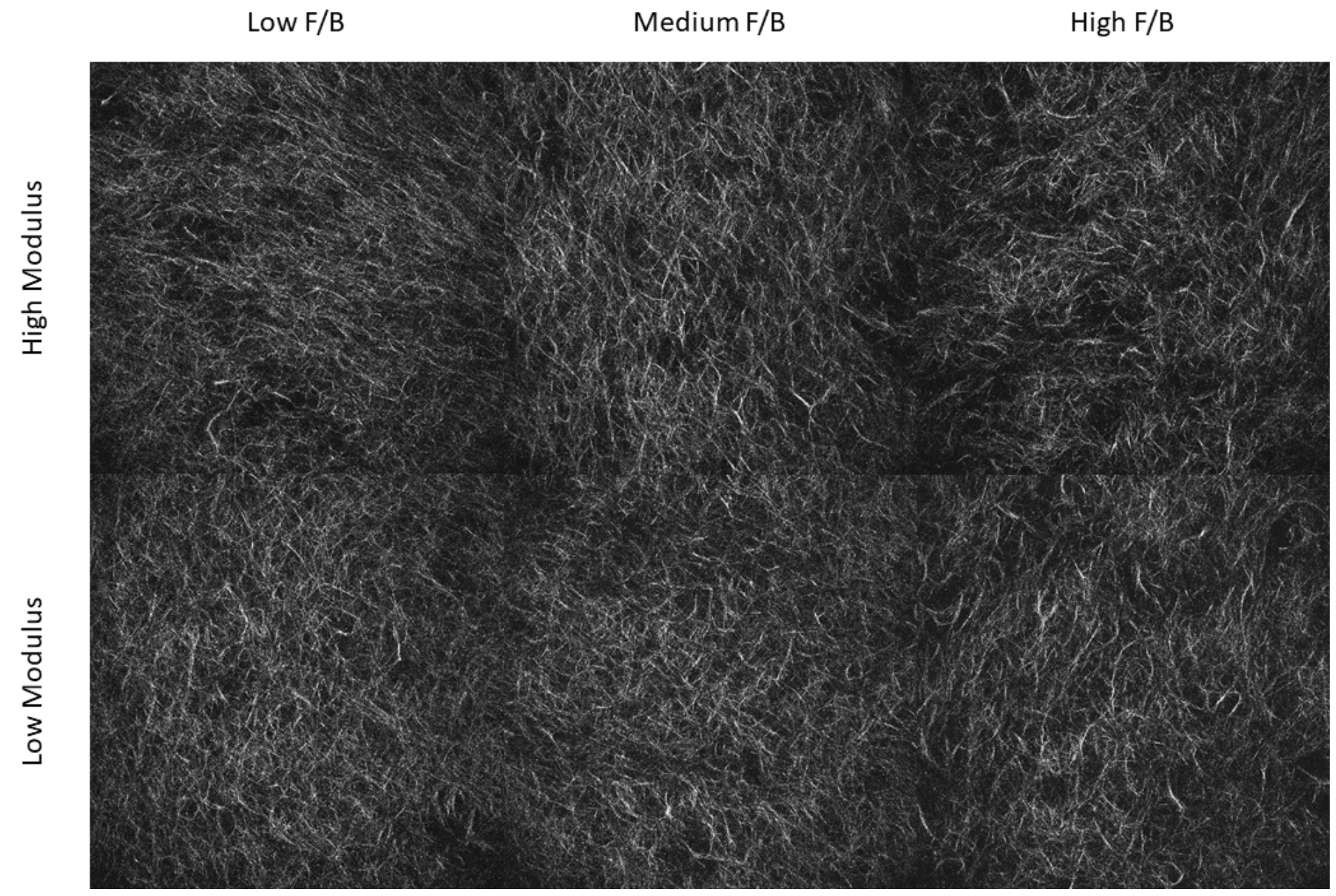

Figure 1

Forward-scattered SHG images of mixed gels at each combination of F/B and elastic modulus. Images are 660 microns across. 


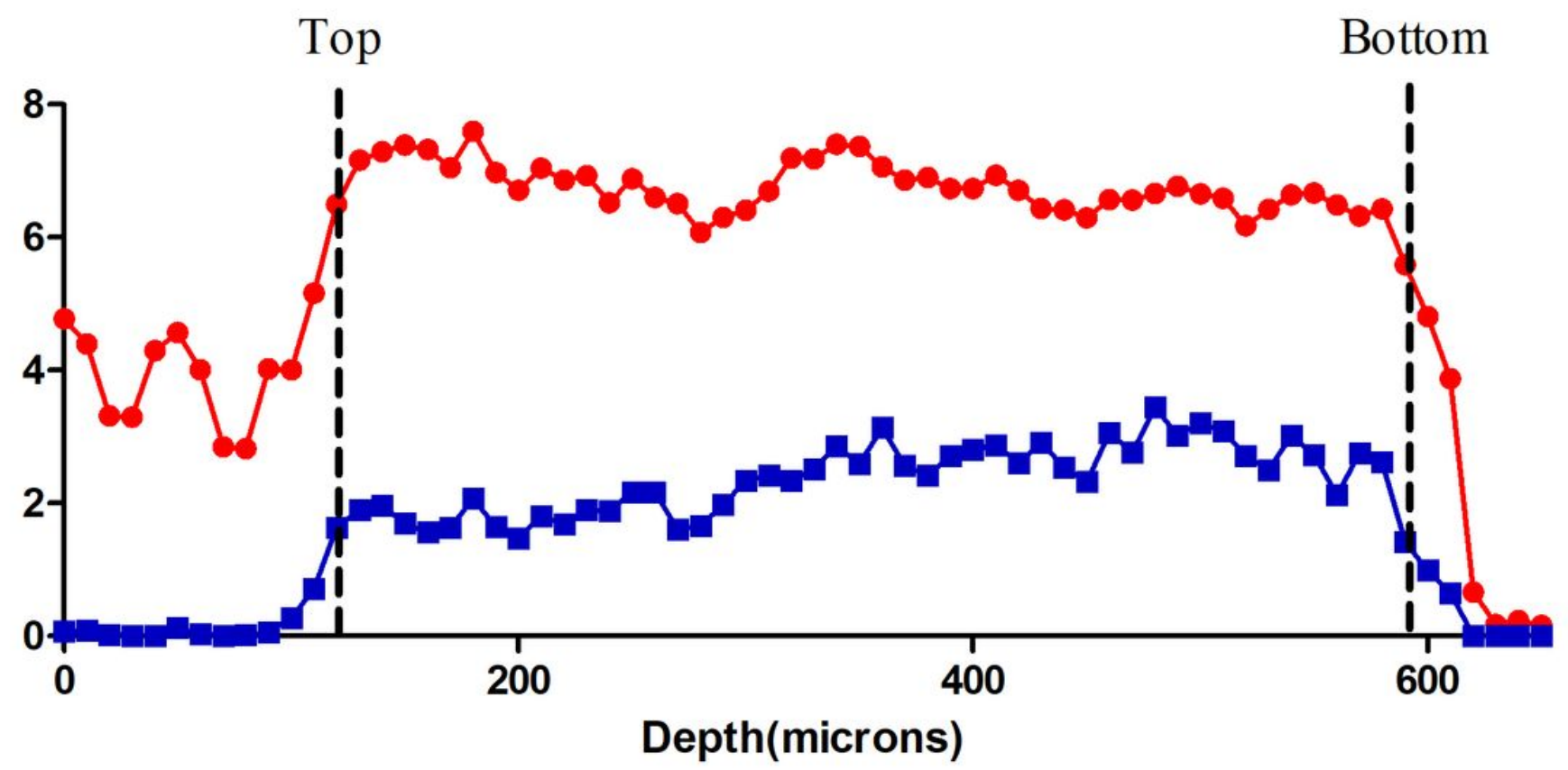

$\rightarrow F / B$

$\rightarrow \%$ pixels above threshold

Figure 2

F/B as a function of depth into the gel. In these gels, F/B (red) fluctuates but does not evidence a significant trend over the depth of the gel. The percentage of pixels above threshold (blue) provides an indication of where the image plane is within the gel. Note that image planes which intersect little or no gel had a very low \% of pixels above threshold but nonetheless had an F/B value, representing the F/B of a few noise pixels or a stray fiber above the gel. 


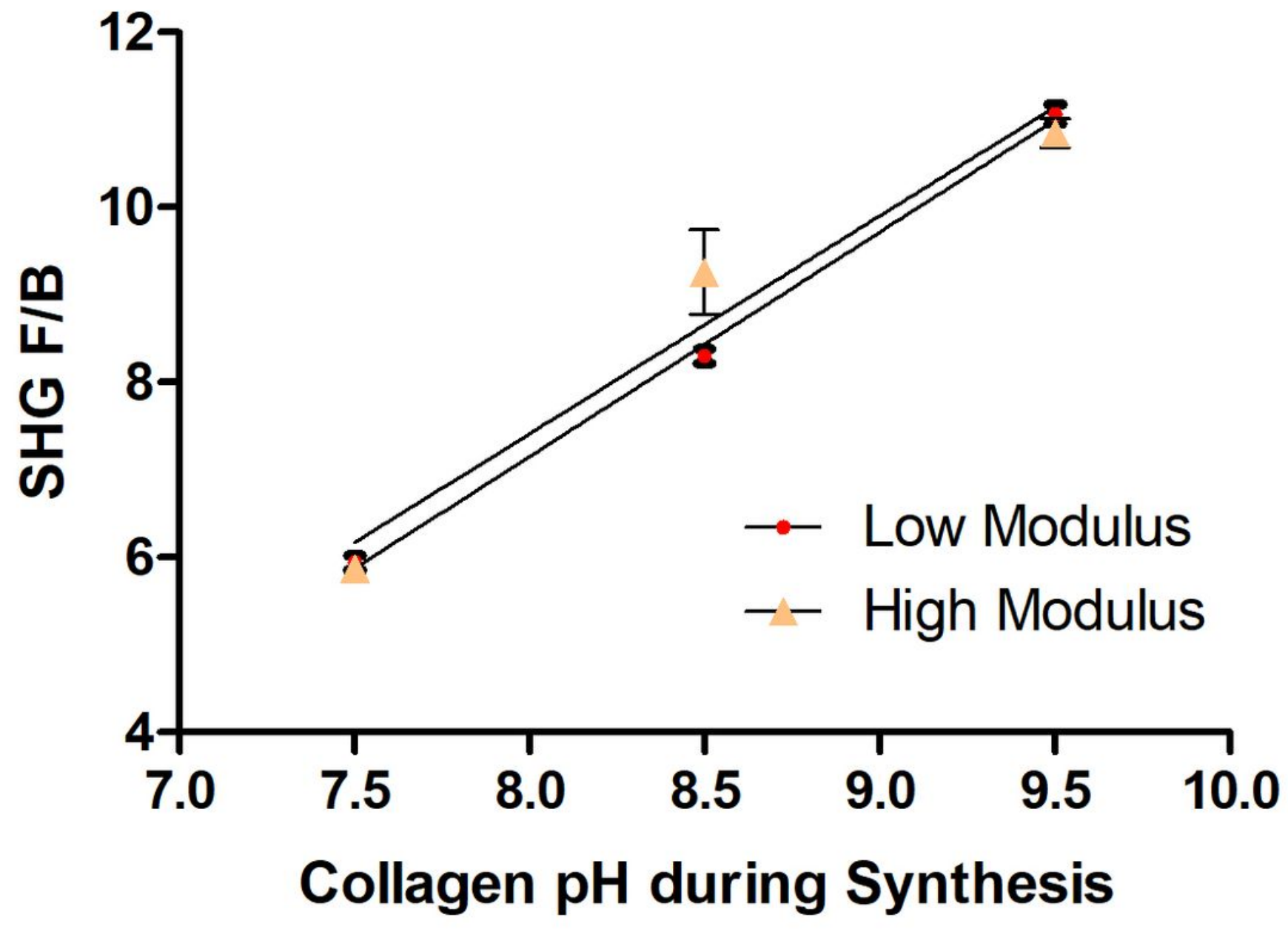

Figure 3

Relationship between SHG F/B and the collagen $\mathrm{pH}$ during synthesis. Each point represents $\mathrm{n}=3$ gels where each gel value is an average of the F/B of three different regions of that gel. Data shown as averages \pm SEM. 


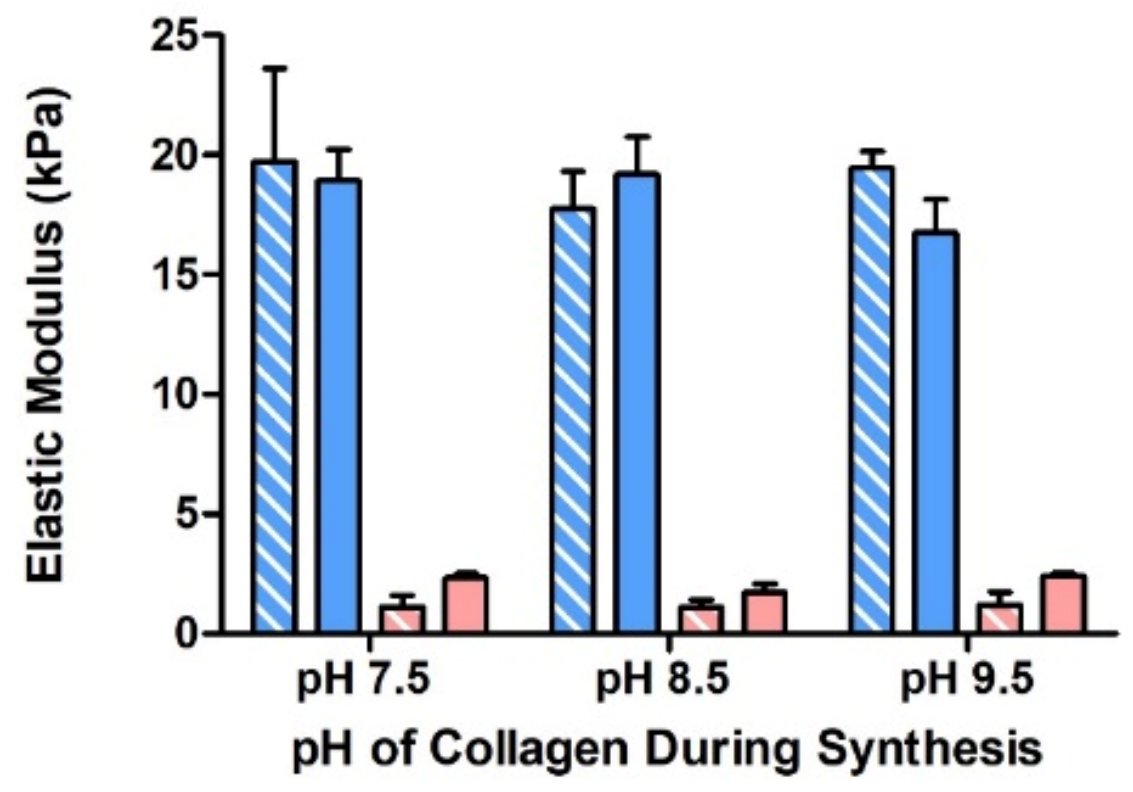

$\square$ AFM: High Modulus

$\square$ Rheometer: High Modulus

$\square$ AFM: Low Modulus

$\square$ Rheometer: Low Modulus

Figure 4

AFM ( $n=3)$ and rheometer $(n=5)$ measurement of 'high' and 'low' modulus gels of various synthesis conditions. Data shown as averages \pm SEM.
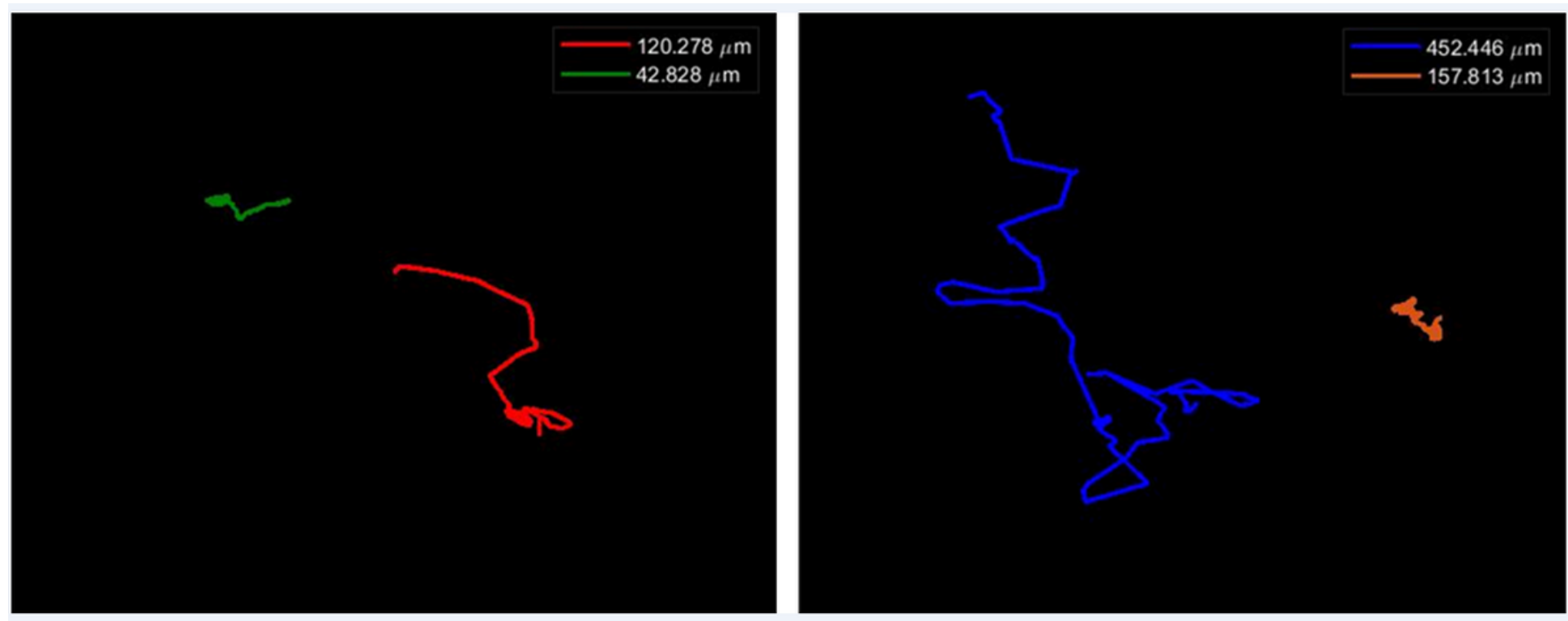

Figure 5

Sample traces of cell motility of two 67NR (left) and two 4T1 (right) cells on high elastic modulus, high F/B gels. For clarity, the two images are shown at different magnifications: the total distance traveled by each cell is recorded near each trace. 

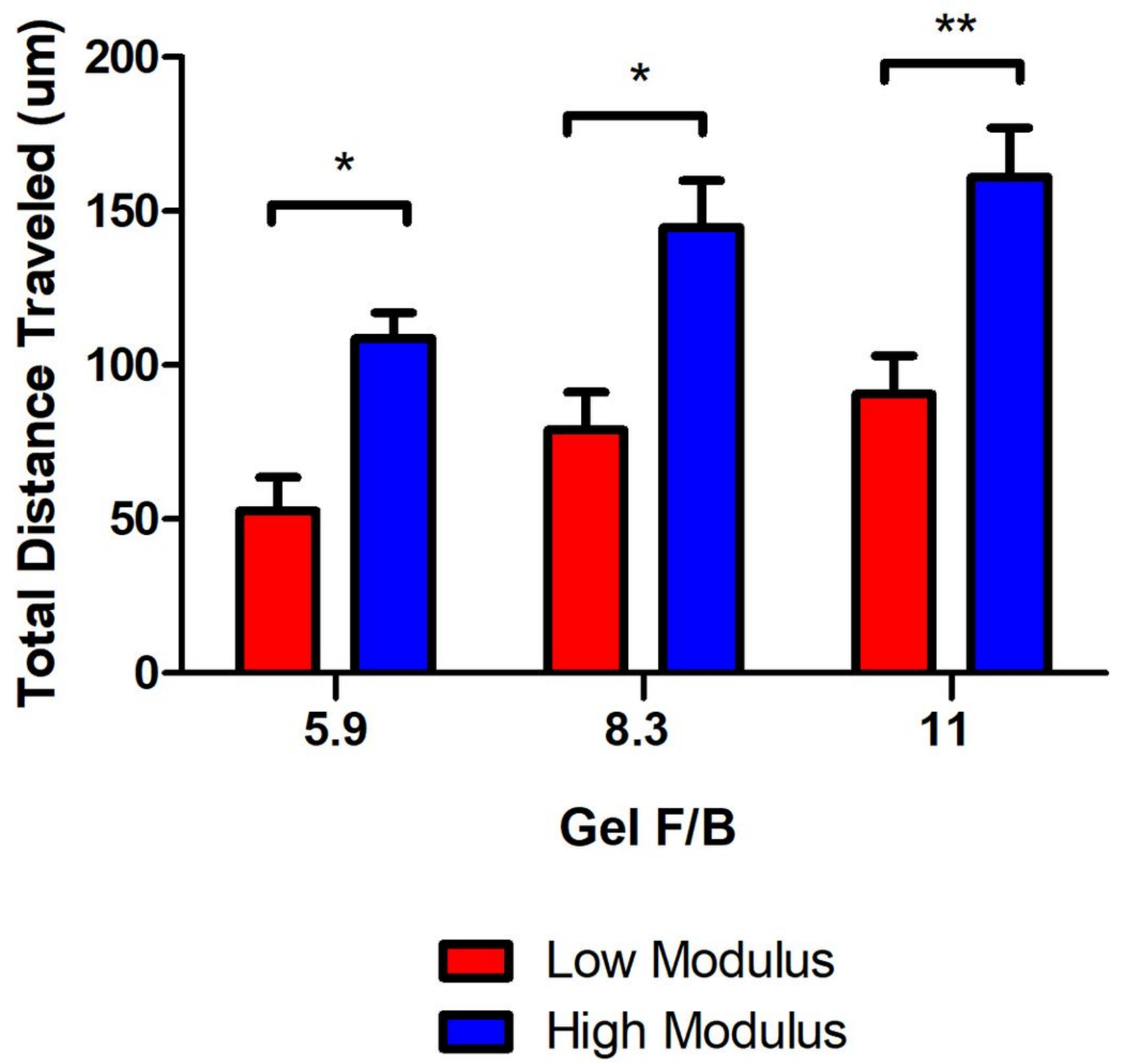

Figure 6

Effect of collagen fiber internal structure, as reported by $\mathrm{F} / \mathrm{B}$, and elastic modulus on tumor cell motility of 4T1 cells. Data shown as averages \pm SEM ( $n=3$ gels). Red: Total distance traveled on high modulus gels. Blue: Total distance traveled on low modulus gels. 


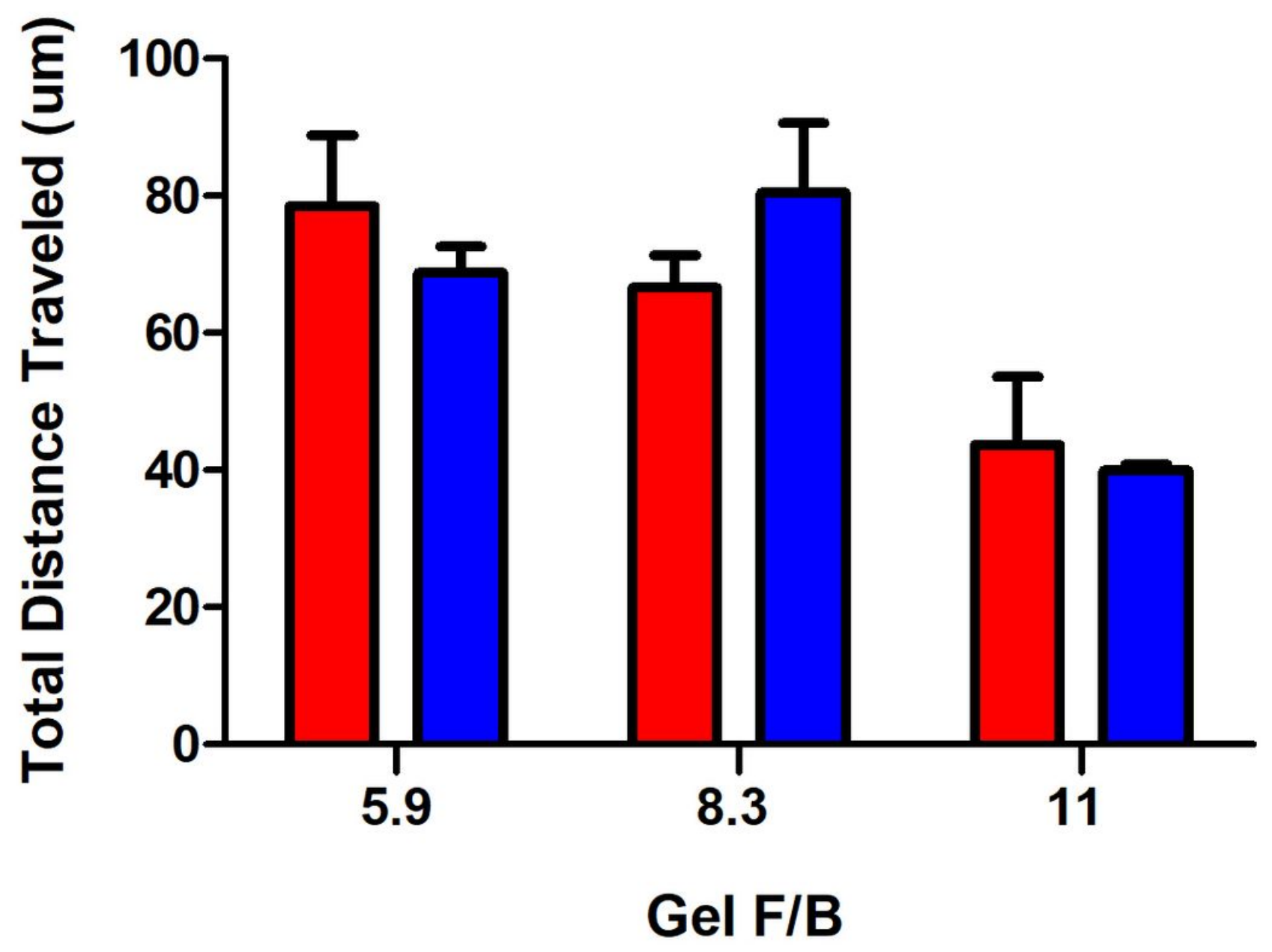

\section{$\square$ Low Modulus High Modulus}

Figure 7

Effect of collagen fiber internal structure, as reported by $\mathrm{F} / \mathrm{B}$, and elastic modulus on tumor cell motility of $67 N R$ cells. Data shown as averages \pm SEM $(n=3)$. Red: Total distance traveled on high modulus gels. Blue: Total distance traveled on low modulus gels.

\section{Supplementary Files}

This is a list of supplementary files associated with this preprint. Click to download.

- 67NRVideo1.gif

- 67NRVideo2.gif

- 4T1Video1.gif 
- 4T1Video2.gif

- SupplementalFigure1.tif

- SupplementalFigure2.jpg

- SupplementalFigure3.tif 